\title{
Near-field coupling and resonant cavity modes in plasmonic
}

\section{nanorod metamaterials}

Haojie Song ${ }^{1}$, Junxi Zhang ${ }^{1,2}$, Guangtao Fei $^{1}$, Junfeng Wang $^{1}$, Kang Jiang ${ }^{3}$, Pei Wang ${ }^{3}$, Yonghua Lu ${ }^{3}$, Ivan Iorsh ${ }^{4}$, Wei Xu ${ }^{1}$, Junhui Jia ${ }^{1}$, Lide Zhang ${ }^{1}$, Yuri S. Kivshar ${ }^{2,4,5}$ and Lin Zhang ${ }^{2}$

${ }^{1}$ Key Laboratory of Materials Physics and Anhui Key Laboratory of Nanomaterials and Nanostructures, Institute of Solid State Physics, Chinese Academy of Sciences, Hefei 230031, P. R. China

${ }^{2}$ Aston Institute of Photonic Technologies (AIPT), School of Engineering \& Applied Science, Aston University, Birmingham B4 7ET, UK

${ }^{3}$ Department of Optics and Optical Engineering, University of Science and Technology of China, Hefei 230026, P. R. China

${ }^{4}$ ITMO University, St. Petersburg 197101, Russia

${ }^{5}$ Nonlinear Physics Centre, Research School of Physics and Engineering, Australian National University, Canberra ACT 2601, Australia

To whom correspondence should be addressed. E-mail: jxzhang@issp.ac.cn 


\begin{abstract}
Plasmonic resonant cavities are capable of confining light at the nanoscale, resulting in both enhanced local electromagnetic fields and lower mode volumes. However, conventional plasmonic resonant cavities possess large Ohmic losses at metal-dielectric interfaces. Plasmonic near-field coupling plays a key role in a design of photonic components based on the resonant cavities because of a possibility to reduce losses. Here, we study the plasmonic near-field coupling in the silver nanorod metamaterials treated as resonant nanostructured optical cavities. Reflectance measurements reveal the existence of multiple resonance modes of the nanorod metamaterials being consistent with our theoretical analysis. Furthermore, our numerical simulations show that the electric field at the longitudinal resonances forms standing waves in the nanocavities due to the near-field coupling between the adjacent nanorods, and a new hybrid mode emerges due to a coupling between nanorods and a gold-film substrate. We demonstrate that this coupling can be controlled by changing a gap between the silver nanorod arrays and gold substrate.
\end{abstract}

Keywords: plasmonic resonance, cavity, near-field coupling, resonance modes, nanorod arrays, silver nanorods, metamaterials 


\section{Introduction}

Control over the light-matter interactions at the nanoscale is one of the main goals at the way towards the realization of photonic integrated circuits [1]. One of the main figure of merit for the light-matter interactions in the Purcell factor - the rate of the spontaneous emission of the light source normalized to that rate in vacuum. According to the seminal paper [2] the Purcell factor can be estimated as the ratio of the cavity quality factor $\mathrm{Q}$ and the electromagnetic mode volume $\mathrm{V}$. Thus, in order to enhance light-matter interaction and thus increase the Purcell factor, we can either increase our cavity quality factor, or decrease its volume. The first approach is widely used in all-dielectric resonant cavities, such as optical microcavities (Fabry-Pérot (F-P), whispering gallery and photonic crystal cavities) [3], silicon-based metasurface analogues of electromagnetically induced transparency (EIT) [4], and layered patch arrays [5]. At the same time, all-dielectric cavities have limitations on a minimal size and thus it is difficult to squeeze light to deeply subwavelength volumes except for a few of particular designs [4].

The alternative approach relies on the utilization of the plasmonic nanocavities which are conventionally characterized by lower quality factors due to the large Ohmic losses but are advantageous over their all-dielectric counter-parts since they allow to localize the electromagnetic modes at the nanometer scale [6-9], thus achieving unprecedented local electromagnetic field amplitude enhancement [10-13].

In the last decade the research interest to the plasmonic nanostructures was constantly growing due to their promising applications in highly integrated optical devices, such as laser [1, 11], multi-channel wavelength demultiplexer [14], slow light systems [15], plasmonic filters [16], plasmonic waveguide systems [17], optical switching [18], broadband solar perfect absorbers [19], and solar desalination devices [20]. It has been noted that plasmonic mode coupling plays a key role in the functional applications of the plasmonic nanostructures based on the near-field interactions between metal-dielectric interfaces, since the plasmonic mode coupling between the different interfaces can decrease the Ohmic losses and thus 
increase the quality factor. For instance, a quality factor up to 330 can be achieved based on squeezing the LSPR band of a gold nanorod into an ultra-narrow single resonance band by coupling it with a whispering gallery cavity of an optical microfiber [21]. Similarly, a strong coupling also appears between a twodimensional plasmonic crystal metasurface and photonic cavity modes [22]. On the other hand, metalinsulator-metal (MIM) waveguides can allow optical mode volumes to be reduced to deeply subwavelength scales with minimal field decay out of the waveguide physical cross section even for frequencies far from the plasmon resonance [23]. Moreover, the interactions between two or more plasmonic structures gives rise to the hybridization of the plasmonic modes [24-26] and strong optical field coupling [27], which can be utilized to tune the plasmonic properties of the systems. Namely, twodimensional arrays of plasmonic rods exhibit strong near field interactions and large Purcell factor enhancement [1]. However, the vertical structures require complex fabrication and are less amenable to large area substrates. In addition, current reports on the vertical nanostructure arrays are mostly focused on gold nanostructures due to their higher stability $[17,18,23-25]$ as compared to the silver nanostructures, since silver-based systems suffer from the oxidization which quenches their plasmon excitation. At the same time the silver is more favorable in terms of plasmonic applications since it is characterized by lower Ohmic losses and larger absolute value of real part of dielectric permittivity in the visible frequency range. This results in the lower decay rates and stronger field localization of the plasmonic modes in silver nanostructures as compared to gold. Therefore, obtaining the silver nanostructures with higher stability remains a fundamental challenge of major importance for the plasmonic technology.

Here we report the plasmonic near-field coupling by using tunable plasmonic resonant nanocavities based on silver nanorod metamaterials, which consist of hexagonally packed arrays of silver nanorod arrays embedded in anodic aluminum oxide (AAO). Through tuning the structural parameters of the resonant nanocavities, light can couple strongly with surface plasmons producing multiple resonance 
modes dependent on the parameters of the nanostructures. Moreover, we show that open plasmonic resonant nanocavities [28, 29] based upon the silver nanorod arrays can be realized by removing the substrate. These open nanocavities based on the plasmonic metamaterials have a plethora of perspective applications such as label-free biosensors with efficient sensing due to enhanced electric fields in an open cavities [29-32], plasmonic nanolasers with enhanced stimulated emission processes based on optical amplification of band-edge lattice plasmons [1] and others. Main advantage of the open nanocavity structures is that they can be easily transferred to other target substrates. In this work we also explore the strong coupling between the substrate surface plasmons and the nanorod structure eigenmodes showing the emergence of the new type of hybrid plasmonic eigenmodes.

\section{Experimental section}

\subsection{Synthesis of anodic aluminum oxide (AAO) membranes}

AAO membranes were fabricated by a two-step anodization method. First, High-purity aluminum sheets ( $99.999 \%, 0.3 \mathrm{~mm}$ thick) were degreased in acetone, and then an annealing treatment under vacuum $\left(10^{-}\right.$ ${ }^{4} \mathrm{~Pa}$ ) was carried out at $500{ }^{\circ} \mathrm{C}$ for $5 \mathrm{~h}$ to remove the mechanical stress and make the grains grow up. The aluminum sheets were electropolished at $23 \mathrm{~V}$ in a 1:9 mixture solution of per chloric acid and ethanol at $2{ }^{\circ} \mathrm{C}$. Then, the aluminum sheets were anodized under a constant voltage of $45 \mathrm{~V}$ in a $0.3 \mathrm{M}$ oxalic acid solution at $3{ }^{\circ} \mathrm{C}$ for $10 \mathrm{~h}$. After that, the anodic oxide layers formed on the sheets were removed in a mixture solution of $6 \% \mathrm{H}_{3} \mathrm{PO}_{4}$ and $1.8 \% \mathrm{H}_{2} \mathrm{CrO}_{4}$ at $60{ }^{\circ} \mathrm{C}$. Subsequently, those sheets were correspondingly anodized again under the constant voltage of $45 \mathrm{~V}$ in the $0.3 \mathrm{M}$ oxalic acid solution at 5 ${ }^{\circ} \mathrm{C}$ for $10 \mathrm{~h}$. After, the aluminum substrates were removed in a $\mathrm{SnCl}_{4}$ solution, the through-channel AAO membranes were achieved by a chemical etching treatment in a $5 \% \mathrm{H}_{3} \mathrm{PO}_{4}$ solution at $30{ }^{\circ} \mathrm{C}$. Finally, the nanochannel sizes of the AAO membranes were tuned by the etching duration time. 


\subsection{Preparation of silver nanorod metamaterials}

Silver nanorod arrays with uniform sizes were prepared by using a galvanostatic electrodeposition into the nanochannels of self-ordered AAO membranes. First, a gold layer with about $100 \mathrm{~nm}$ thickness was sputtered onto the bottom surface of the AAO membranes to serve as a working electrode, the graphite was used as a counter electrode. Then, the silver nanorods were directly electrodeposited into the nanochannels of the AAO templates at a constant potential of $-0.1 \mathrm{~V}$ Vs counter electrode at $15^{\circ} \mathrm{C}$, using an electrolyte containing $\mathrm{AgNO}_{3}(10 \mathrm{~g} \mathrm{~L} 1)$, EDTA (5 g L1), $\mathrm{Na}_{2} \mathrm{SO}_{3}(50 \mathrm{~g} \mathrm{L1})$, and $\mathrm{K}_{2} \mathrm{HPO}_{4}(20 \mathrm{~g} \mathrm{L1})$, the silver nanorods were not oxidized by using the chelating agent EDTA in the electrolyte solution to decrease the reducing potential of the $\mathrm{Ag}^{+}$as well as enhance the oxidation potential of $\mathrm{OH}^{-}$to produce $\mathrm{O}_{2}$ during the electrochemical process. As a result, the lower potential resulted in growth of the Ag nanorods but avoided the oxidation of Ag during the growth. In addition, the Ag nanorods were embedded in the AAO membranes, which also prevent oxidation of the Ag nanorods. The diameter of the silver nanorods was controlled by the nanochannel diameters of the AAO membranes, and the length of the silver nanorods was adjusted by the electrodeposition time from $45 \mathrm{~s}(150 \mathrm{~nm}), 100 \mathrm{~s}(280 \mathrm{~nm}), 200 \mathrm{~s}$ $(350 \mathrm{~nm})$ to $300 \mathrm{~s}(420 \mathrm{~nm})$. Finally, the gold layer was completely removed by a fine polishing method, and the silver nanorods embedded in AAO membranes with transparent deep yellow color were obtained.

\subsection{Characterization and optical measurements of silver nanorod metamaterials}

The silver nanorod arrays embedded in the AAO membranes were characterized by field-emission scanning electron microscopy (FE-SEM, Sirion 200). Reflectance measurements of the silver nanorod arrays were performed on the UV-Vis-NIR optical spectrometer with a fixed-angle reflector $\left(8^{\circ}\right)$ and calibrated against a diffuse reflectance module. 


\section{Results and discussion}

Figure 1 shows scanning electron microscopy (SEM) images of silver nanorod metamaterials. There are two configurations of the structures: in the first configuration the nanorods stand on the gold substrate (right side of figure 1(a), right), and in the second configuration the gold substrate is removed, the system thus forming an open plasmonic cavity (figure 1(a), left). The top view of the SEM image displays the silver nanorod metamaterial consisting of the hexagonally packed nanorod array embedded in an anodic aluminum oxide (AAO) membrane. Figures $1 \mathrm{~b}$ and $1 \mathrm{c}$ display the cross-section images of the silver nanorod arrays with the substrate of gold film and without the substrate, respectively, figures $1 \mathrm{~d}$ and $1 \mathrm{e}$ correspond the schematic illustrations of the nanorod arrays with and without the substrate. The nanorod length can be tuned from $150 \mathrm{~nm}$ to $420 \mathrm{~nm}$ by varying the electrodeposition time.

The plasmonic properties of the silver nanorod metamaterials are explored based on the reflection spectra measured by use of a UV-Vis-NIR optical spectrometer with a fixed-angle reflector $\left(8^{\circ}\right)$ and calibrated against a diffuse reflectance module. Figure 2 shows the reflectance spectra of the open nanocavity structure. For the silver nanorod arrays with perfect alignment and periodicities, light can couple strongly with surface plasmons to produce characteristic dips in the reflectance at specific angles of incidence defined by the size and the structure of the material. The dispersion of the resonance modes of the plasmonic cavity can be tailored by the nanorod length. The peaks marked with $\mathrm{T}$ are the transversal resonance mode of surface plasmons at around $360 \mathrm{~nm}$. Furthermore, the peaks marked with $\mathrm{L}_{\mathrm{i}}(\mathrm{i}=1,2$, 3 and 4) represent the longitudinal resonance modes with different orders of surface plasmons, which are strongly dependent on the length of the nanorods. When the nanorod length increases from $150 \mathrm{~nm}$ to 420 $\mathrm{nm}$, high-order harmonic modes of the longitudinal resonances are observed. The resonance mode L1 appears at the wavelength of $740 \mathrm{~nm}$ for the nanorod length of $150 \mathrm{~nm}$ (Figure 2(a)). As the nanorod length increases to $280 \mathrm{~nm}$, two longitudinal resonance modes L1 and L2 are observed at $1085 \mathrm{~nm}$ and $624 \mathrm{~nm}$, respectively (Figure 2(b)). Three longitudinal resonance modes L1, L2 and L3 appear around 
$1115 \mathrm{~nm}, 640 \mathrm{~nm}$ and $519 \mathrm{~nm}$, respectively when the length of the silver nanorod reaches $350 \mathrm{~nm}$ (Figure 2(c)). Four longitudinal resonance modes L1, L2, L3 and L4 are found at $1500 \mathrm{~nm}, 860 \mathrm{~nm}, 602 \mathrm{~nm}$ and $522 \mathrm{~nm}$ as the nanorod length increases to $420 \mathrm{~nm}$ (Figure 2(d)).

These experimental results are generally consistent with those of the theoretical simulations (the red dotted curves) by the following Finite-difference time-domain (FDTD) method. A difference in the reflectance spectra observed in experiment and simulations may be attributed to the fact that the silver nanorods are assumed to have perfectly smooth surfaces in the simulations. In fact, the surfaces of the fabricated silver nanorods are not very smooth, and the nanorod length does not very uniformly, so some scattering losses at the surfaces of the nanorods are inevitable. On the other hand, in our work, the reflection spectra are as typical examples to explore the plasmonic resonant cavity modes, this is because the mode characteristics of the high-order resonances can be observed clearly in the reflectance spectra, as well as the measurement method to the high-order longitudinal resonance modes is basically based on the reflection spectra measured by use of the optical spectrometer with the reflectance module. In fact, the transmission spectra and the reflection spectra are complementary each other (see supporting information).

The plasmonic coupling properties of the silver nanorod metamaterials can be simulated by using the FDTD method with Lumerical package. The dispersion model of silver is based on data from Johnson and Christy in the material library of the software [33], the effective refractive index of the AAO membranes is 1.6 considering the simplified calculation [34]. Figure 3(a) shows a FDTD simulation domain for modeling the plasmonic cavity based on hexagonally packed silver nanorods with under periodic boundary conditions, where the period of the nanorod arrays $D_{\text {int }}$ is $115 \mathrm{~nm}$, and the nanorod diameter $2 \mathrm{r}$ is $66 \mathrm{~nm}$. Perfectly matched layer (PML) boundary conditions in the direction of $z$ and periodic boundary conditions in the $(\mathrm{x}, \mathrm{y})$ plane were used in the simulation code. The order and the wavelength of the longitudinal resonance modes obtained via the FDTD simulation agree with those of the experimental measurements. 
The FDTD simulations are allowed to obtain the electric field $(|E|)$ profiles of the specific resonance modes. Namely, it has been revealed that the electric field of the longitudinal resonance modes with higher-orders are localized at the interface between the silver nanorods and the aluminum oxide, and the electric fields at the interface form a standing-wave mode (Figures. 3(b) and 3(c)). These standing waves originate both from the Fabry-Pérot (F-P) resonance between the terminations of the silver nanorods and from plasmonic coupling between the adjacent nanorods in the periodic MIM (Ag nanorod-Aluminum oxide-Ag nanorod) interfaces. Figure 3(b) demonstrates the electric field distributions of the standing waves with multiple orders (e.g., L1, L2, L3, and L4) in the silver nanorod arrays with the nanorod length of $420 \mathrm{~nm}$, which correspond to the longitudinal resonance at $1500 \mathrm{~nm}, 860 \mathrm{~nm}, 623 \mathrm{~nm}$, and $522 \mathrm{~nm}$, respectively. It is observed that there are two half-antinodes in the mode L1, which appear at the two ends of the silver nanorods for the open plasmonic cavity. The L1 mode thus supports half period phase change along the nanorod length. Generally, the $i$-th longitudinal mode acquires the $\pi i$ phase along the nanorods. According to the electric field distributions of the silver nanorod metamaterials with the different longitudinal modes of LSPs, we thus can write down an analogue of the condition for the longitudinal waves in the nanorod metamaterial

$$
\frac{i}{2} \lambda_{i}=l
$$

Where $i$ (e.g., 1, 2, 3 and 4) is the order of the harmonics, $\lambda_{i}$ is the wavelength of the harmonics, $l$ is the length of the open nanocavity (e.g., the nanorod length). Moreover, the wavevector $k_{i}$ and the length of the nanocavity have the form according to the half-wave mode

$$
k_{i}=\frac{i \pi}{l}
$$

It is interesting to note that the higher order harmonic modes in the open plasmonic nanocavity based upon the silver nanorod metamaterials are similar to the half-wave harmonics produced within the acoustic 
cavities of certain types of musical instruments [35]. These standing waves with half-wave modes are different from those in the semi-open nanocavity structures mentioned below.

Furthermore, the reflectance spectra and the electric field distributions of the open plasmonic nanocavities with different periods of the silver nanorod arrays are simulated by the FDTD method. It is found that the longitudinal resonance mode of surface plasmons present obvious blue-shifts with increasing the periods of the silver nanorod from $90 \mathrm{~nm}$ to $115 \mathrm{~nm}$ (Figure 4(a)), which originates from the different plasmonic coupling between the adjacent nanorods for the different spacing of the nanorods. Moreover, the electric fields enhance when the period of the nanorod arrays decreases from $115 \mathrm{~nm}$ to 90 nm (Figures. 4(b) to 4(d)) since the coupling interaction between the adjacent nanorods becomes stronger.

Moreover, the dispersion relation is assumed to be valid for all values of $l$ when the other structural parameters such as the diameter-spacing ratio of the nanorods and the refractive index between the nanorods arrays are fixed. Figure 5 illustrates the dispersion relation of the nanorod arrays with increasing the nanorod length. The experiment data shown in figure 2 match the dispersion relation perfectly, indicating that the standing wave of the nanorod cavity comply with the dispersion relation for all the nanorod lengths when the other structure parameters are unchanged. The light line in the AAO plotted in figure 5, illustrates that the standing wave of surface plasmons are bound in the cavity and have a bigger wavevector than light. It is found that the different orders show the different area in the dispersion relation, for example, the first order mode occupies the area of lower energy. The standing wave in the silver nanorod metamaterials generated by the coupling between nanorods is similar to that of the optical antenna [36-38].

The plasmonic properties display sharp differences between an open cavity and a semi-open cavity. Figures 6(a) and 6(b) illustrate the reflectance spectra of the silver nanorod arrays without and with the gold film substrate. It can be seen that the substrate of gold film has a remarkable influence on the resonance modes of the subwavelength plasmonic cavity based on the Ag nanorod arrays (Figure 6(a)). 
Compared with the resonance modes of the open plasmonic cavity, the resonance peaks with different harmonic modes exhibit pronounced blue-shifts. The distribution of electromagnetic field of open cavity is symmetrical in the interior of the cavity, and that is asymmetric in the semi-open cavity (Figure 6(b)). The near field coupling exhibits another way to tune the resonance modes of surface plasmons [39-41]. It is important to note that the substrate of the gold film may narrow the bandwidth of the standing-wave.

Since the gold film substrate have a significant impact on the resonance modes of surface plasmons of the silver nanorod arrays, the plasmonic near-field coupling between the open-cavity of the silver nanorod arrays and the gold substrate were explored further by the FDTD simulation (Figure 7). In the calculations, we selected the length, the period, and the diameter of the silver nanorods to be $150 \mathrm{~nm}, 115 \mathrm{~nm}$ and 66 $\mathrm{nm}$, respectively, for the gold film of $50 \mathrm{~nm}$ in thickness as the substrate. In figure 7 it can be seen that the resonance mode (e.g., the dotted line) displays a blue-shift compared with that shown in figure 2(a). Moreover, if the aluminum oxide spacer is introduced, a new resonance mode was found, which can be defined a plasmonic coupling resonance mode. Additionally, we can see the coupling resonance mode between the silver nanorod arrays and the gold substrate performs a blue-shift when the gap increases (Figure 7(a)). This is quite opposite to the red-shift observed in the resonance modes of the coupled planar uniform plasmonic interfaces $[38,42]$. This can be explained the unconventional nanorod resonance mode dispersion by wave dispersion relation, according to the dispersion curve of the gap between the metal films, the dispersion relation of the gap approaches to that of light when the gap increases, that is to say, the resonance frequency increase for the same momentum. Since the momentum depends on the period of the nanorod arrays, for the nanorod arrays and metal film, the coupling system consisting of the silver nanorod arrays and the gold film has the same momentum due to the fixed period in the arrays, as a result, the resonance mode blue-shifts with increasing the gap due to the enhancement of the resonance frequency. 
On the other hand, the coupling resonance mode between the silver nanorod arrays and the gold substrate is strongly dependent on the gap. Namely, it is observed that the local field of the plasmonic nanocavity enhance with the increase of the gap from $2 \mathrm{~nm}$ to $25 \mathrm{~nm}$ (Figure $7 \mathrm{~b}$ ). We observe the transition from the strong to the weak coupling between the nanorod cavity resonance mode and the surface plasmon when the gap increases.

\section{Conclusion}

In summary, we have studied, both experimentally and theoretically, plasmonic properties of resonant nanocavities in silver nanorod metamaterials. Reflectance measurements suggest that open resonant nanocavities exhibit multiple plasmonic resonances, which can be tailored by the length of the silver nanorods. Both order and wavelength of the longitudinal resonance modes obtained by the FDTD simulation are consistent with the experimental data. Furthermore, we have observed that the electromagnetic fields of the higher-order resonance modes are focused at the interface between silver nanorods and aluminum oxide, and the electric fields at the interface form standing waves, which indicates that the silver nanorod metamaterials can work as plasmonic cavity resonators. An open plasmonic nanocavity exhibits sharp half-wave harmonic resonances with the symmetric distribution of electromagnetic field in the interior of the cavities. We have predicted and observed a new hybrid mode which results from the coupling of silver nanorod arrays with a gold substrate, and the resonance of the plasmonic coupling mode of the silver nanorod arrays gradually become weaker as the gap increases.

\section{Acknowledgements}

This work was supported by National Natural Science Foundation of China (Grant No. 10804112), National Basic Research Program of China (Grant No. 2012CB932303, 2012CB921900), Seventh Framework Program of the European Union for Research for the Marie Curie International Fellowship 
(FP7-PEOPLE-2013-IIF, Grant No. 623473), Australian Research Council, and Visiting Professorship Program of the Leverhulme Trust.

\section{References}

[1] Zhou W, Dridi M, Suh J Y, Kim C H, Co D T, Wasielewski M R, Schatz G C and Odom T W 2013 Nat. Nanotechnol. 8 506-11

[2] Purcell E M 1946 Phys. Rev. 69 681-81

[3] Vahala K J 2003 Nature 424 839-46

[4] Yang Y, Kravchenko I I, Briggs D P and Valentine J 2014 Nature Commun. 55753

[5] Liu X, Liu G, Fu G, Liu M and Liu Z 2016 Nanotechnology 27125202

[6] Sorger V J, Ye Z, Oulton R F, Wang Y, Bartal G, Yin X and Zhang X 2011 Nat. Commun. 2331

[7] Oulton R F, Sorger V J, Zentgraf T, Ma R M, Gladden C, Dai L, Bartal G and Zhang X 2009 Nature $461629-32$

[8] Akimov A V, Mukherjee A, Yu C L, Chang D E, Zibrov A S, Hemmer P R, Park H and Lukin M D 2007 Nature $450402-6$

[9] de Leon N P, Shields B J, Yu C L, Englund D E, Akimov A V, Lukin M D and Park H 2012 Phys. Rev. Lett. 108, 226803

[10] Zhang J and Zhang L 2012 Adv. Opt. Photon. 4 157-321

[11] Zhang W, Ding F, Li W D, Wang Y, Hu J and Chou S Y 2012 Nanotechnology 23225301

[12] Hofmann C E, de Abajo F J and Atwater H A 2011 Nano Lett. 11 372-6

[13] Gramotnev D K and Bozhevolnyi S I 2010 Nat. Photon. 4 83-91

[14] Wang G, Lu H, Liu X, Mao D and Duan L 2011 Opt. Express 19 3513-8

[15] Zhang S, Genov D A, Wang Y, Liu M and Zhang X 2008 Phys. Rev. Lett. 101047401

[16] Si G, Zhao Y, Lv J, Lu M, Wang F, Liu H, Xiang N, Huang T J, Danner A J, Teng J and Liu Y J 2013 
Nanoscale 5 6243-8

[17] Dai D and He S 2009 Opt. Express 17 16646-53

[18] Krasavin A V and Zayats A V 2010 Appl. Phys. Lett. 97041107

[19] Liu Z, Liu X, Huang S, Pan P, Chen J, Liu G and Gu G 2015 Acs Appl. Mater. Interfaces 7 4962-8

[20] Zhou L, Tan Y, Wang J, Xu W, Yuan Y, Cai W, Zhu S and Zhu J 2016 Nature Photon. 10 393-9

[21] Wang P, Wang Y, Yang Z, Guo X, Lin X, Yu X-C, Xiao Y-F, Fang W, Zhang L, Lu G, Gong Q and Tong L 2015 Nano Lett. 15 7581-6

[22] Liu Z, Liu G, Liu X, Huang S, Wang Y, Pan P and Liu M 2015 Nanotechnology 26235702

[23] Jung J, Sondergaard T and Bozhevolnyi S I 2009 Phys. Rev. B 79035401

[24] Verellen N, Sonnefraud Y, Sobhani H, Hao F, Moshchalkov V V, Van Dorpe P, Nordlander P and Maier S A 2009 Nano Lett. 9 1663-7

[25] Lassiter J B, Sobhani H, Fan J A, Kundu J, Capasso F, Nordlander P and Halas N J 2010 Nano Lett. $103184-9$

[26] Prodan E, Radloff C, Halas N J and Nordlander P 2003 Science 302 419-22

[27] Liu Z, Shao H, Liu G, Liu X, Zhou H, Hu Y, Zhang X, Cai Z and Gu G 2014 Appl. Phys. Lett. 104 081116

[28] Lyvers D P, Moon J-M, Kildishev A V, Shalaev V M and Wei A 2008 Acs Nano 2 2569-76

[29] Simovski C R, Belov P A, Atrashchenko A V and Kivshar Y S 2012 Adv. Mater. 24 4229-48

[30] Kabashin A V, Evans P, Pastkovsky S, Hendren W, Wurtz G A, Atkinson R, Pollard R, Podolskiy V A and Zayats A V 2009 Nat. Mater. 8 867-71

[31] Vigderman L, Khanal B P and Zubarev E R 2012 Adv. Mater. 24 4811-41

[32] Liu Z, Liu G, Liu X, Fu G, Liu M and 2014 IEEE Photon. Tech. Lett. 26 2111-4

[33] Lumerical FDTD Solutions, Available from: http://www.lumerical.com/

[34] Eriksson T S, Hjortsberg A, Niklasson G A and Granqvist C G 1981 Appl. Opt. 20 2742-6 
[35] Patterson R D, Smith D R R, van Dinther R and Walters T C 2008 Auditory perception of sound sources Springer p. 43.

[36] Arango F B, Kwadrin A and Koenderink A F 2012 ACS Nano 6 10156-67

[37] Boriskina S V and Dal Negro L 2010 Opt. Lett. 35 538-40

[38] Kurokawa Y and Miyazaki H T 2007 Phys. Rev. B 75035411

[39] Chu H S, Ewe W B, Koh W S and Li E P 2008 Appl. Phys. Lett. 92103103

[40] Halterman K, Elson J M and Singh S 2005 Phys. Rev. B 72075429

[41] Kottmann J and Martin O 2001 Opt. Express 8 655-63

[42] Belov P A, Dubrovka R, Iorsh I, Yagupov I and Kivshar Y S 2013 Appl. Phys. Lett. 103161103 


\section{Figure captions}

Figure 1. FE-SEM images of silver nanorod arrays embedded in AAO membranes. (a) Top views of the silver nanorod arrays, where left side shows the nanorods after removal of a gold film substrate, the right side corresponds the nanorod arrays standing on the substrate; $(b, c)$ cross-section views of the silver nanorod arrays with and without the substrate, respectively, $(\mathrm{d}, \mathrm{e})$ schematic illustration of the structures in $(b, c)$.

Figure 2. UV-Vis-NIR reflectance spectra of silver nanorod arrays embedded in AAO membranes for the nanorod length of (a-d) $150 \mathrm{~nm}, 280 \mathrm{~nm}, 350 \mathrm{~nm}$ and $420 \mathrm{~nm}$, respectively. Red dotted curves are the results of the FDTD simulations, and black solid curves correspond to the experimental data.

Figure 3. (a) FDTD simulation domain for modeling the plasmonic cavity responses of silver nanorods with an arrangement a 2D hexegonal lattice under periodic boundary conditions. $D_{\text {int }}$ is the period of the nanorod arrays (e.g., $115 \mathrm{~nm}$ ), $2 \mathrm{r}$ denotes the nanorod diameter (e.g., $66 \mathrm{~nm}$ ), $\mathrm{h}$ is the nanorod length. (b) Electric field $(|E|)$ distributions of the silver nanorod metamaterials with the different longitudinal modes of LSPs for the same nanorods of $420 \mathrm{~nm}$ in length. (c) Electric field (|E|) distributions of the silver nanorod metamaterials with the same longitudinal modes of the first order in the silver nanorod arrays for the different nanorod lengths.

Figure 4. (a) Reflection spectra of the silver nanorod arrays with the different periods of $90 \mathrm{~nm}, 100 \mathrm{~nm}$ and $115 \mathrm{~nm}$ by the FDTD simulations. (b), (c) and (d) correspond the electric field ( $|E|)$ distributions of the silver nanorod metamaterials with the different periods when the length and the diameter are fixed. 
Figure 5. Dispersion relation for the standing-wave modes $(i=1,2)$ of silver nanorod arrays in AAO $\left(D_{\text {int }}\right.$ $=115 \mathrm{~nm}, 2 \mathrm{r}=66 \mathrm{~nm}, \mathrm{n}=1.6$ ), compared with ligh line in AAO (red dash line), distinct points were ploted as experiment data ( $i$ stand for the order), the black solid line represents the dispersion relation of the nanorods array for the standing-wave modes.

Figure 6. (a) Experimental reflection spectra of silver nanorod arrays embedded in AAO, the black solid curve corresponds to the experimental results of the open plasmonic nanocavity, the red dotted curve represents the semi-open plasmonic nanocavity. (b) Simulated Electric field (|E|) distribution of the first order mode (L1) of longitudinal resonance of LSPs in the silver nanorod arrays.

Figure 7. Simulated reflectance spectra (a) and the electric field $(|E|)$ distributions (b) of the open plasmonic nanocavities with different gaps between the silver nanorod arrays and the gold film as a substrate, the thickness of the gold film is $50 \mathrm{~nm}$, and the dotted line represents the $0 \mathrm{~nm}$ gap as semi-open nanocavity, the gap changes from $2 \mathrm{~nm}$ to $25 \mathrm{~nm}$. 
(a)

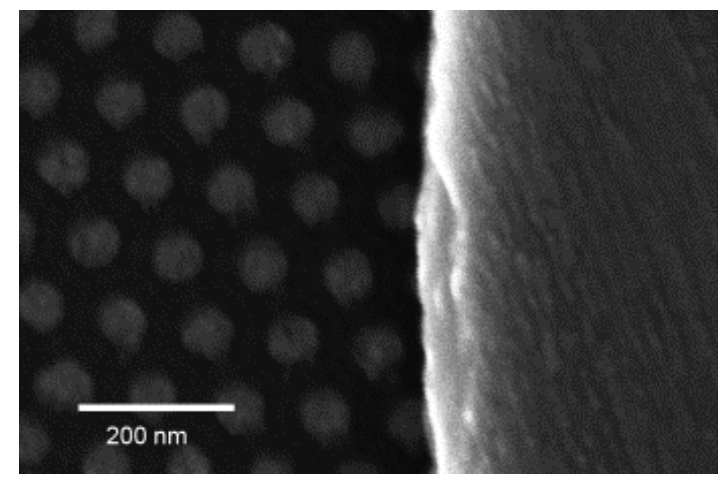

(b)
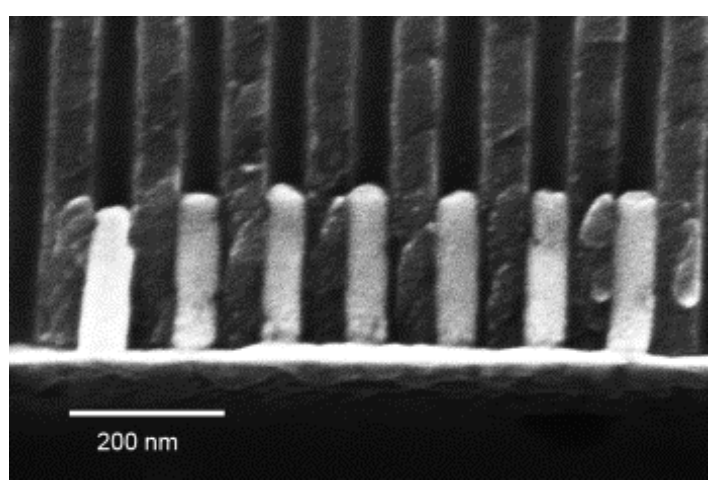

(c)

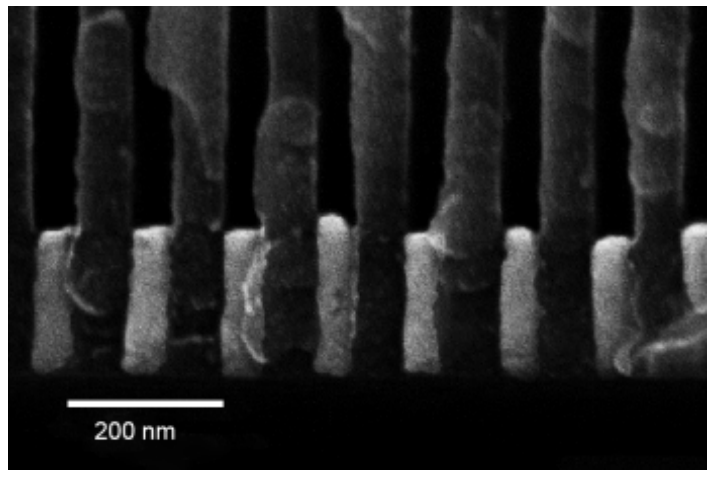

(d)

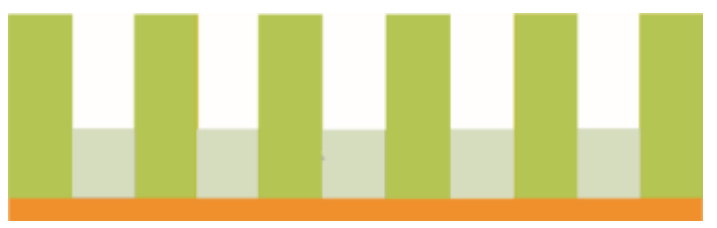

(e)

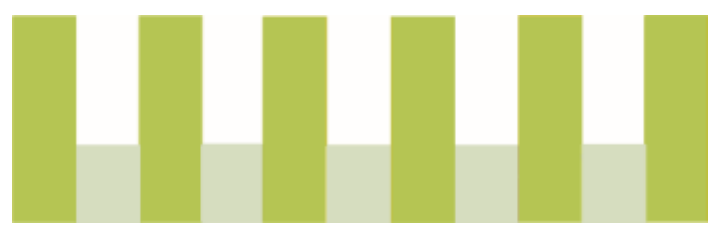

Figure 1 
(a)

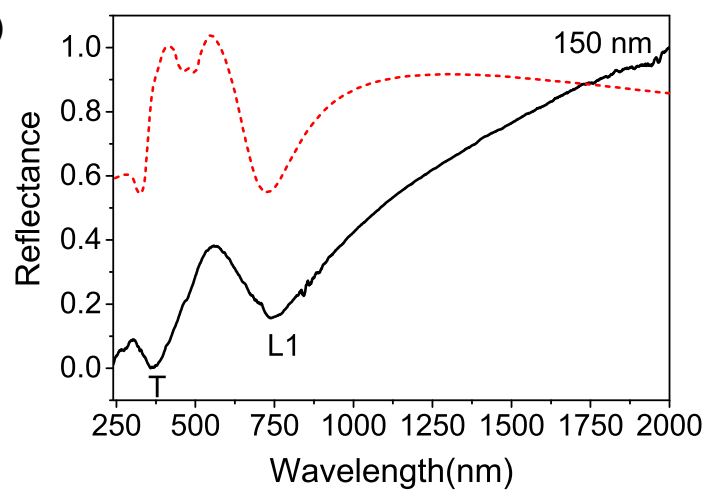

(b)

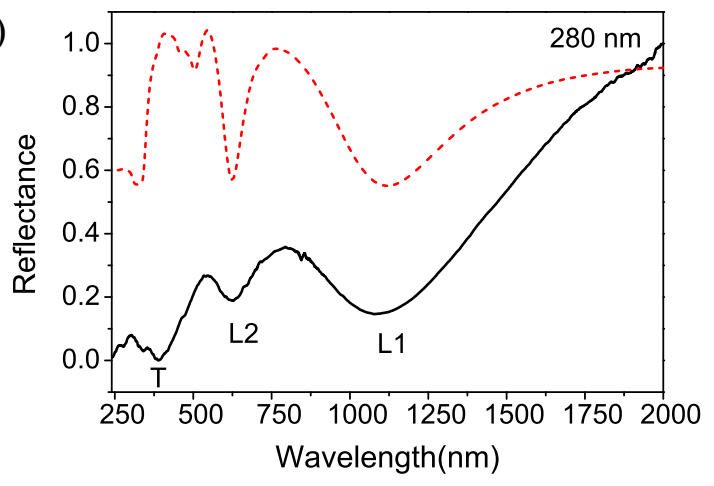

(c)

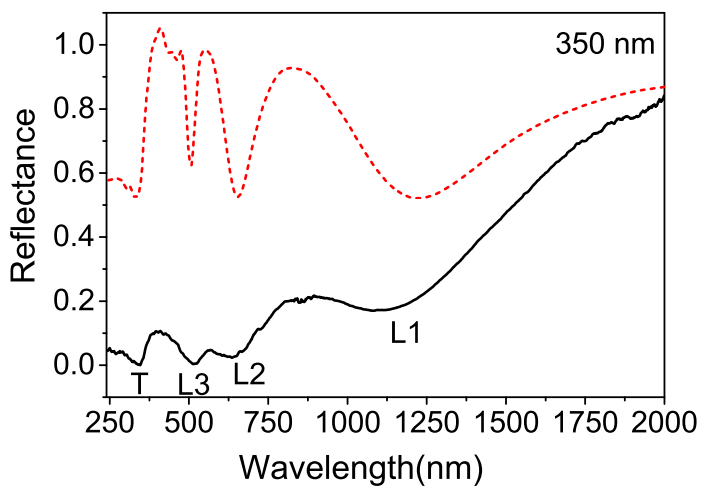

(d)

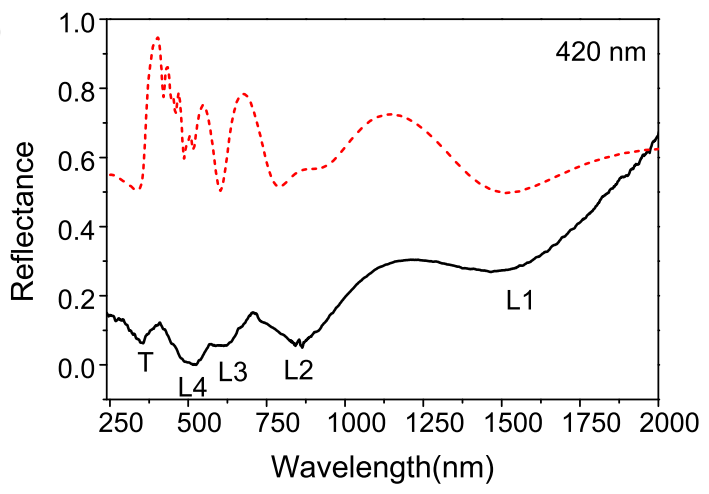

Figure 2 
(a)

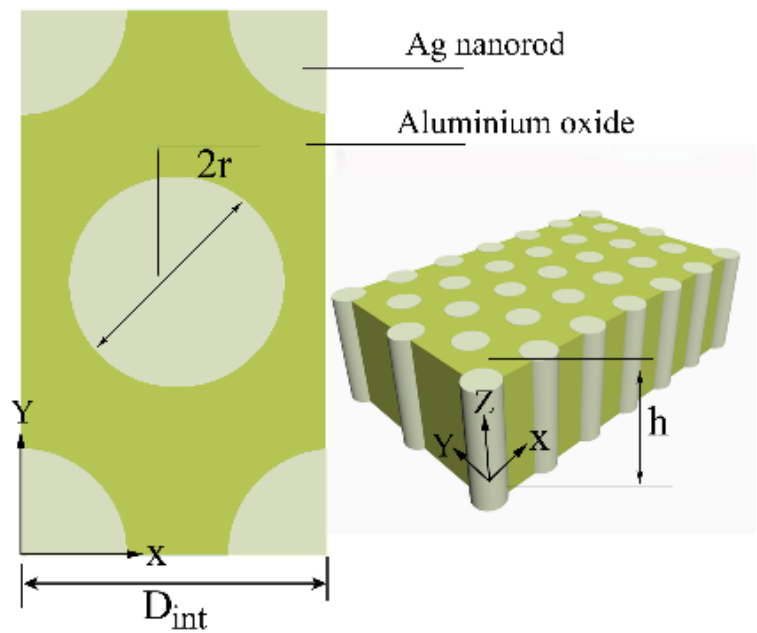

(b)

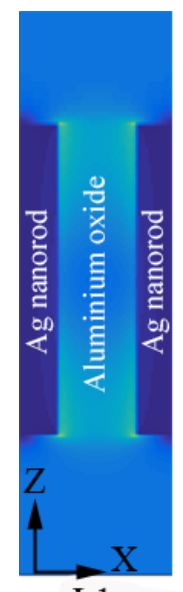

L1

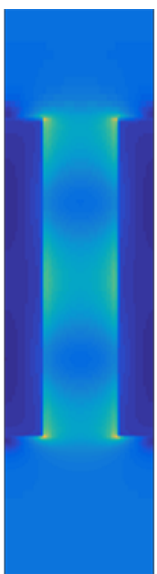

L2

(c)

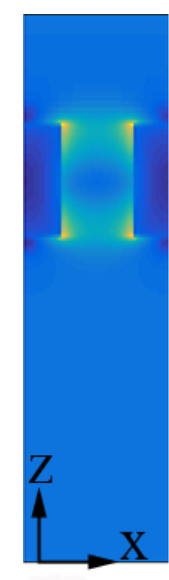

$150 \mathrm{~nm}$

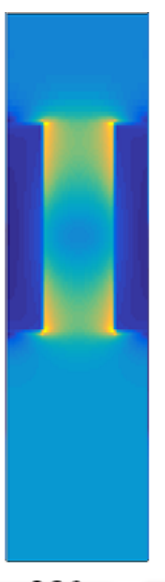

$280 \mathrm{~nm}$

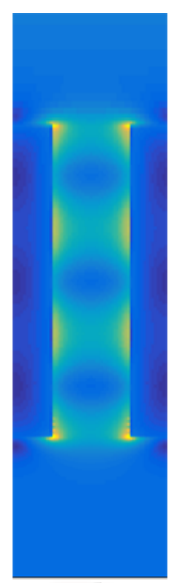

L3
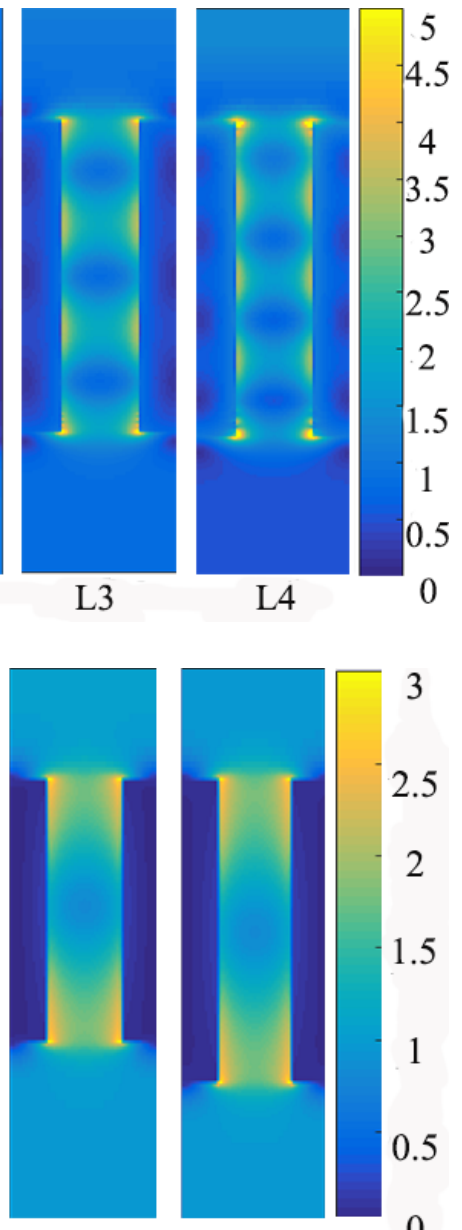

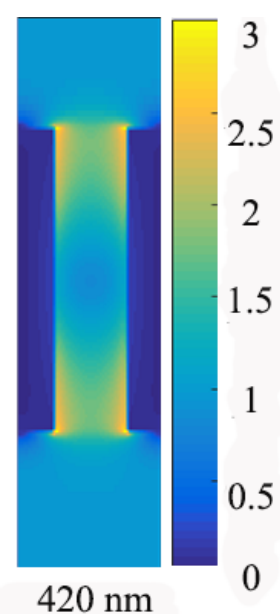

1.5

0.5
$350 \mathrm{~nm}$

Figure 3 

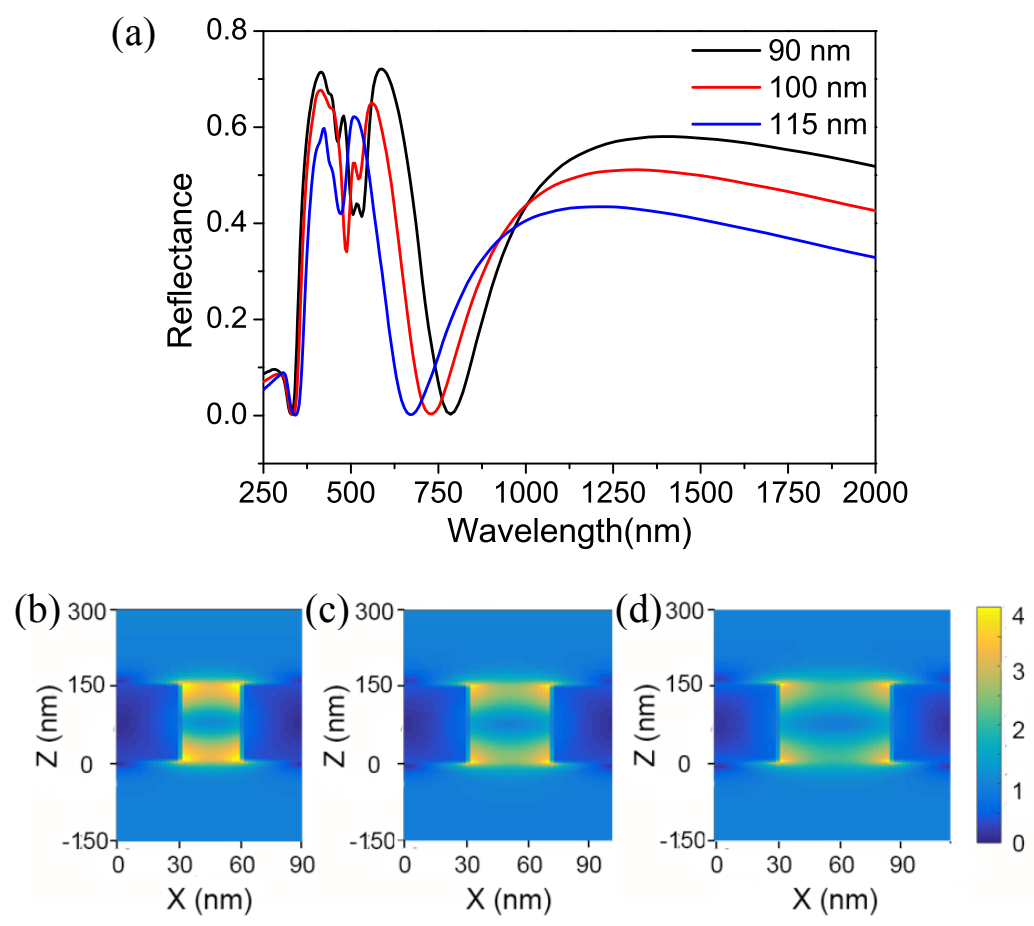

Figure 4 


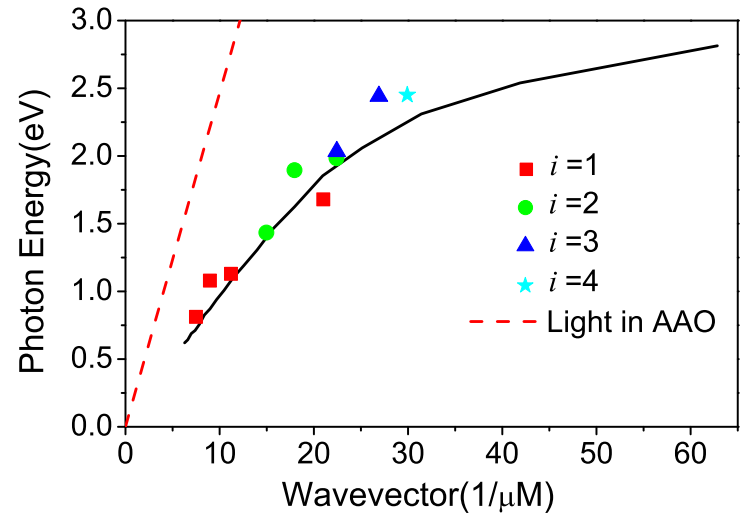

Figure 5 
(a)

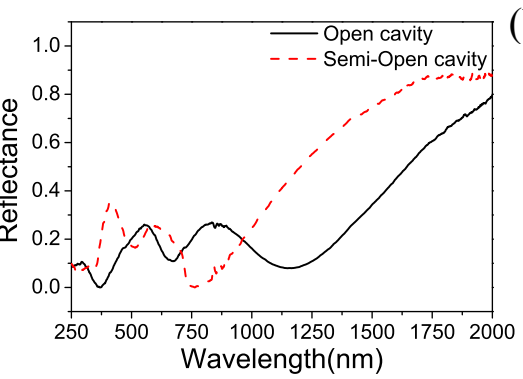

(b)

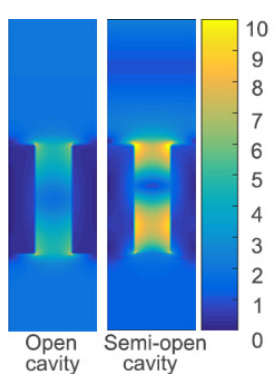

Figure 6 
(a)

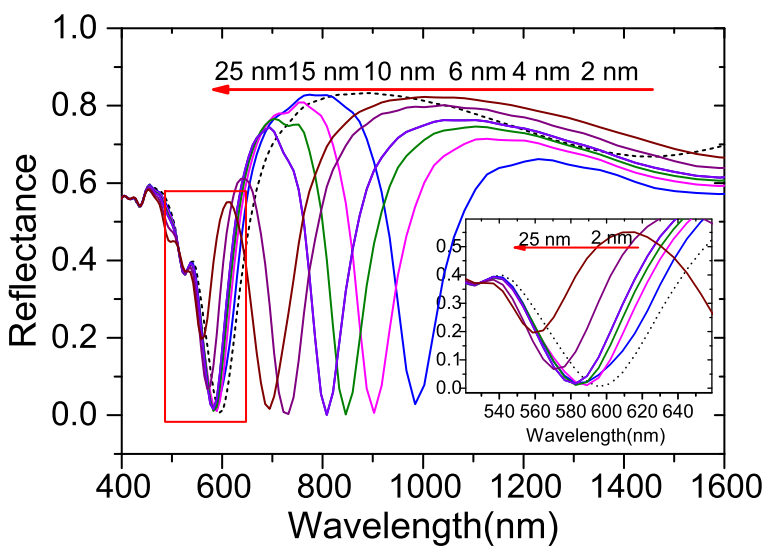

(b)

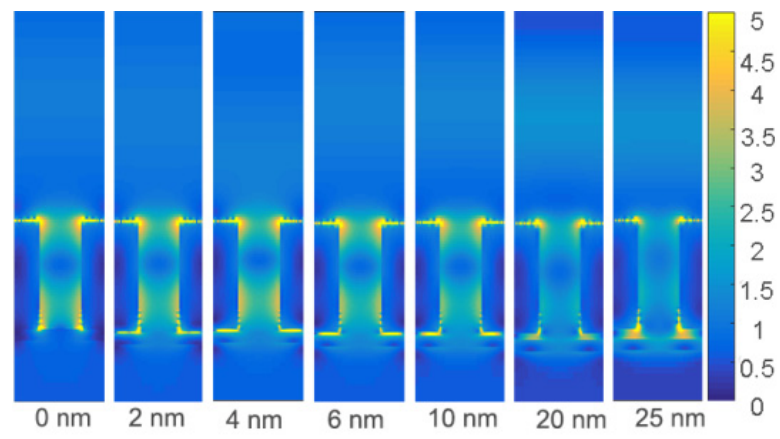

Figure 7 